\title{
Decrease in the number of patients diagnosed with cancer during the COVID-19 pandemic in Germany
}

\author{
Louis Jacob ${ }^{1,2}$ (1) $\cdot$ Matthias Kalder ${ }^{3} \cdot$ Karel Kostev $^{4}$ (D)
}

Received: 20 November 2021 / Accepted: 11 January 2022 / Published online: 18 January 2022

(c) The Author(s), under exclusive licence to Springer-Verlag GmbH Germany, part of Springer Nature 2022

\begin{abstract}
Purpose Little is known on how coronavirus disease 2019 (COVID-19) has impacted cancer diagnosis in Germany since the first lockdown in March 2020. Therefore, this retrospective study aimed to compare the number of patients newly diagnosed with cancer in general and specialized practices in Germany between April 2020-March 2021 and April 2019-March 2020. Methods Patients aged $\geq 18$ years with at least 1 visit to 1 of 1403 general and specialized practices in Germany in April 2020-March $2021(n=3,804,596)$ and April 2019-March $2020(n=3,913,386)$ were included in this retrospective study. Specialized practices were composed of gynecology, dermatology and urology practices. Cancer diagnoses included all types of cancer documented using the International Classification of Diseases, 10th revision (ICD-10 codes: C00-C97). The number of patients newly diagnosed with cancer per practice was compared between April 2020-March 2021 and April 2019-March 2020 using Wilcoxon tests.

Results There were 126,379 and 138,996 patients diagnosed with cancer in April 2020-March 2021 and April 2019-March 2020 , respectively. The number of patients diagnosed with cancer decreased in all types of practice, and this decrease was significant in general practices $(-7.1 \%, p$ value $=0.038)$. In terms of cancer type, this decrease was particularly pronounced for skin cancers $(-12.8 \%, p$ value $=0.025)$.

Conclusion The COVID-19 pandemic has been associated with a decrease in the number of patients newly diagnosed with cancer in general and specialized practices in Germany. Public health interventions are urgently warranted to mitigate the deleterious effects of this health crisis on cancer diagnosis.
\end{abstract}

Keywords COVID-19 pandemic $\cdot$ Cancer diagnosis $\cdot$ General practices $\cdot$ Specialized practices $\cdot$ Germany

\section{Introduction}

Coronavirus disease 2019 (COVID-19) is a viral disorder caused by the severe acute respiratory syndrome coronavirus 2 (SARS-CoV-2) (Wiersinga et al. 2020). COVID-19

Karel Kostev

karel.kostev@iqvia.com

1 Research and Development Unit, Parc Sanitari Sant Joan de Déu/CIBERSAM, Dr. Antoni Pujadas, 42, Sant Boi de Llobregat, Barcelona, Spain

2 Faculty of Medicine, University of Versailles Saint-Quentin-en-Yvelines, 78180 Montigny-le-Bretonneux, France

3 Department of Gynecology and Obstetrics, Philipps University of Marburg, 35037 Marburg, Germany

4 Department of Epidemiology, IQVIA, Unterschweinstiege 2-14, 60549 Frankfurt am Main, Germany symptoms include pulmonary (e.g., cough, shortness of breath and sputum production) and extra-pulmonary manifestations (e.g., abnormal heart rhythm, headache and diarrhea) (Johnson et al. 2020). As of December 23, 2021, more than 276 million people have contracted the COVID-19 in the world, while the number of related deaths has exceeded 5.3 million (World Health Organization 2021). In Germany, the respective figures are 6.9 million for the number of positive cases and 109,700 for the number of deaths (World Health Organization 2021).

The COVID-19 pandemic has negatively impacted the diagnosis and the management of several conditions of potential life-threatening nature. For example, a study, using data from the French national hospitalization database, revealed that hospital admissions for myocardial infarction decreased by $31 \%$ after the beginning of the lockdown in March 2020 in France (Lantelme et al. 2020). It was observed in another study of emergency departments 
from the United States that daily stroke alerts had significantly decreased since the beginning of the COVID-19 era (Uchino et al. 2020). The deleterious effects of the COVID19 pandemic on the care of acute and chronic disorders may be explained, at least partially, by overwhelmed healthcare systems (Tangcharoensathien et al. 2021) and the fear of patients to consult primary care practices and emergency departments due to the potential risk of being infected by the SARS-CoV-2 (Czeisler et al. 2020; Lazzerini et al. 2020). Some literature also suggests that COVID-19 has been associated with a substantial decrease in cancer diagnosis (Andrew et al. 2021; De Luca et al. 2021; Erdmann et al. 2021; Gurney et al. 2021; Jacob et al. 2021; Kuzuu et al. 2021; Patt et al. 2020; Peacock et al. 2021; Piontek et al. 2021; Reichardt et al. 2021; Ruiz-Medina et al. 2021; Stang et al. 2020; Tsibulak et al. 2020; Vardhanabhuti and $\mathrm{Ng}$ 2021; Voigtländer et al. 2021). Interestingly, a study conducted in Germany found that cancer diagnoses decreased in March-May 2020 compared with March-May 2019, and this decrease was particularly strong for cancers of the skin and the respiratory and intrathoracic organs (Jacob et al. 2021). Although the findings of this study are of particular importance, only the first months of the COVID-19 crisis were analyzed, and it is thus not known if this health crisis had a prolonged deleterious impact on cancer diagnosis in this country. Most of other studies also conducted in Germany have obtained similar results (Erdmann et al. 2021; Piontek et al. 2021; Reichardt et al. 2021; Stang et al. 2020; Voigtländer et al. 2021). However, the majority of these studies included data obtained early in the pandemic and in a single state (e g., Saxony (Piontek et al. 2021), North RhineWestphalia (Stang et al. 2020) or Bavaria (Voigtländer et al. 2021)), limiting the generalizability of their findings to the entire country during the whole pandemic.

Therefore, the goal of this retrospective study was to compare the number of patients newly diagnosed with cancer in general and specialized practices in Germany between April 2020-March 2021 and April 2019-March 2020.

\section{Materials and methods}

\section{Database}

Data from the Disease Analyzer database (IQVIA) were used for this study. This database has already been described in the literature (Rathmann et al. 2018). Briefly, the Disease Analyzer database contained demographic, diagnosis and prescription data anonymously obtained from the computer systems of general and specialized practices in Germany. Diagnosis data are coded using the International Classification of Diseases, 10th revision (ICD-10), while prescription data are coded using the Anatomical Classification of
Pharmaceutical Products of the European Pharmaceutical Marketing Research Association (EphMRA). IQVIA assesses on a regular basis the quality of the data based on several criteria such as completeness of documentation and linkage between diagnoses and prescription. Practices to include in the Disease Analyzer database are selected based on the summary statistics of practices in Germany published by the German Medical Association every year (Bundesärztekammer 2021). These statistics include information on physician's age, specialty group, community size category, and German federal state. At least 30 practices are sampled per specialty (e.g., general, gynecology and dermatology). A standardized interface allows practices to collect data and send these data to IQVIA in an anonymized format. Summary statistics published by the German Medical Association are reassessed every year, while the panel design of the practices included in the database is adjusted on a yearly basis. When a practice ends its collaboration with IQVIA, this practice is replaced by another practice. The Disease Analyzer database covers around 3\% of all practices in Germany, and previous research has found the database to be representative of practices in the country. Concretely, the demographic characteristics (sex, age and region of residence) of the patients included in the Disease Analyzer database are similar to those of the German population. Besides, the incidence and prevalence of major chronic diseases (e.g., cancer, dementia and diabetes) obtained with the Disease Analyzer database are comparable to those obtained with other sources of data from this country (Rathmann et al. 2018).

\section{Study population}

All patients aged $\geq 18$ years with at least 1 visit to 1 of 1403 general and specialized practices in Germany in April 2020-March 2021 and April 2019-March 2020 were included in this retrospective study. Specialized practices were composed of gynecology, dermatology and urology practices. To be included in the study, practices had to deliver data continuously between April 2018 and March 2021 (study period and pre-observational 12 month period). Patients had to have no diagnosis of cancer in their entire medical history prior to the period of interest (April 2020-March 2021 or April 2019-March 2020).

\section{Study variables}

Cancer diagnoses included all types of cancer and corresponded to ICD-10 codes C00-C97. Analyses were also conducted by cancer type: skin (ICD-10: C43 and C44); male genital organs including prostate (ICD-10: C60-C63); breast (ICD-10: C50); urinary tract (ICD-10: C64-C68); digestive organs (ICD-10: C15-C26); lymphoid, hematopoietic, and 
related tissue (ICD-10: C81-C96); female genital organs (ICD-10: C51-C58); respiratory and intrathoracic organs (ICD-10: C30-C39); thyroid and other endocrine glands (ICD-10: C73-C75); lip, oral cavity, and pharynx (ICD-10: C00-C14); and eye, brain and other parts of central nervous system (ICD-10: C69-C72). Finally, demographic variables included sex and age.

\section{Statistical analyses}

The number of patients newly diagnosed with cancer per practice was compared between April 2020-March 2021 and April 2019-March 2020 using Wilcoxon tests. Analyses were stratified by type of practice, demographic characteristics (sex and age) and type of cancer. $p$ values $<0.05$ were considered statistically significant. Analyses were performed using SAS 9.4.

\section{Results}

\section{Practices' and patients' characteristics}

This study included 994 general, 234 gynecology, 91 dermatology, and 84 urology practices. There were 3,804,596 and 3,913,386 patients with at least 1 visit to 1 of these practices in April 2020-March 2021 and April 2019-March 2020, respectively. Among them, 126,379 individuals were diagnosed with cancer in April 2020-March 2021 and 138,996 in April 2019-March 2020 (difference in the proportion of $-6.5 \%$; Table 1). Mean (standard deviation) age of patients diagnosed with cancer was 65.9 (16.0) years in April 2020-March 2021 and 65.6 (15.9) years in April 2019-March 2020. In addition, there were 50.7\% and 50.8\% of women among individuals with cancer during the two respective study periods.

\section{Differences by practice type in the number of patients newly diagnosed with cancer}

Table 1 displays differences by practice type in the number of patients newly diagnosed with cancer per practice between April 2020-March 2021 and April 2019-March 2020. The number of patients newly diagnosed with cancer per practice was lower in April 2020-March 2021 than in April 2019-March 2020 for all types of practice, and this difference was only significant for general practices $(-7.1 \%$, $p$ value $=0.038$.

\section{Differences by sex and age in the number of patients newly diagnosed with cancer}

Table 2 shows differences by sex and age in the number of patients newly diagnosed with cancer per practice between April 2020-March 2021 and April 2019-March 2020. The number of patients newly diagnosed with cancer per practice decreased for the majority of sex and age groups, and this decrease was significant for men aged 41-50 years $(-15.2 \%, p$ value $=0.046)$, men aged $71-80$ years $(-12.3 \%$, $p$ value $=0.044)$, and women aged $71-80$ years $(-10.0 \%, p$ value $=0.016$ ).

\section{Differences by cancer type in the number of patients newly diagnosed with cancer}

Differences by cancer type in the number of patients newly diagnosed with cancer per practice between April 2020-March 2021 and April 2019-March 2020 are displayed in Table 3. The number of patients newly diagnosed with cancer per practice was lower in April 2020-March 2021 than April 2019-March 2020 for all types of cancer, and this difference was significant for skin cancer only $(-12.8 \%, p$ value $=0.025)$.

Table 1 Overall difference in the number of patients newly diagnosed with cancer, and differences by practice type in the number of patients newly diagnosed with cancer per practice in Germany between April 2020-March 2021 and April 2019-March 2020

\begin{tabular}{|c|c|c|c|c|}
\hline Type of practice & April 2020-March 2021 & April 2019-March 2020 & Difference (\%) & $p$ value $^{\mathrm{a}}$ \\
\hline All practices & $126,379(3,804,596)$ & $138,996(3,913,386)$ & -6.5 & \\
\hline General $(n=994)$ & $53.0(46.2)$ & $57.0(53.8)$ & -7.1 & 0.038 \\
\hline Gynecology $(n=234)$ & $41.4(41.9)$ & $45.0(50.6)$ & -7.9 & 0.700 \\
\hline Dermatology $(n=91)$ & $357.2(410.3)$ & $407.4(563.4)$ & -12.3 & 0.360 \\
\hline Urology $(n=84)$ & $232.5(328.0)$ & $248.9(357.5)$ & -6.6 & 0.728 \\
\hline
\end{tabular}

Data are mean (standard deviation) except for all practices \{number of patients newly diagnosed with cancer [total number of patients] ${ }^{\mathrm{a}} p$ values were obtained using Wilcoxon tests 
Table 2 Differences by sex and age in the number of patients newly diagnosed with cancer per practice in Germany between April 2020-March 2021 and April 2019-March 2020

\begin{tabular}{llllll}
\hline Sex & Age group (years) & $\begin{array}{l}\text { April 2020- } \\
\text { March 2021 }\end{array}$ & $\begin{array}{l}\text { April 2019- } \\
\text { March 2020 }\end{array}$ & Difference (\%) & $p$ value \\
\hline Male & $18-40$ & $2.8(14.3)$ & $2.7(14.8)$ & 0.5 & 0.581 \\
& $41-50$ & $2.5(9.3)$ & $3.0(12.3)$ & -15.2 & 0.046 \\
& $51-60$ & $6.8(20.1)$ & $7.6(23.7)$ & -11.1 & 0.062 \\
& $61-70$ & $9.7(23.3)$ & $10.6(26.9)$ & -7.9 & 0.254 \\
& $71-80$ & $11.0(23.1)$ & $12.6(27.4)$ & -12.3 & 0.044 \\
Female & $>80$ & $8.5(15.9)$ & $8.7(16.5)$ & -2.2 & 0.991 \\
& $18-40$ & $3.7(21.9)$ & $4.2(25.7)$ & -12.7 & 0.083 \\
& $41-50$ & $3.7(10.1)$ & $4.4(15.7)$ & -15.2 & 0.113 \\
& $51-60$ & $7.7(16.1)$ & $8.5(21.7)$ & -8.8 & 0.151 \\
& $61-70$ & $8.5(15.4)$ & $9.2(19.1)$ & -7.1 & 0.379 \\
& $71-80$ & $8.8(14.4)$ & $9.7(17.2)$ & -10.0 & 0.016 \\
& $>80$ & $7.8(11.6)$ & $8.1(12.6)$ & -3.4 & 0.908 \\
\hline
\end{tabular}

Data are mean (standard deviation) unless otherwise indicated

$p$ values were obtained using Wilcoxon tests

Table 3 Differences by cancer type in the number of patients newly diagnosed with cancer per practice in Germany between April 2020-March 2021 and April 2019-March 2020

\begin{tabular}{|c|c|c|c|c|}
\hline Cancer type & April 2020-March 2021 & April 2019-March 2020 & Difference $(\%)$ & $p$ value \\
\hline Skin & $30.9(136.6)$ & $35.4(175.4)$ & -12.8 & 0.025 \\
\hline Male genital organs including prostate & $13.0(57.8)$ & $13.9(64.7)$ & -6.3 & 0.228 \\
\hline Breast & $10.2(16.3)$ & $10.8(20.2)$ & -5.2 & 0.674 \\
\hline Urinary tract & $6.7(60.3)$ & $7.0(62.7)$ & -4.9 & 0.413 \\
\hline Digestive organs & $5.5(8.1)$ & $6.0(10.8)$ & -8.5 & 0.058 \\
\hline Lymphoid, hematopoietic and related tissue & $5.4(14.1)$ & $5.8(16.9)$ & -7.3 & 0.220 \\
\hline Female genital organs & $3.1(9.8)$ & $3.3(11.9)$ & -4.7 & 0.153 \\
\hline Respiratory and intrathoracic organs & $2.3(3.3)$ & $2.5(3.7)$ & -7.4 & 0.329 \\
\hline Thyroid and other endocrine glands & $0.7(1.9)$ & $0.8(1.9)$ & -5.0 & 0.187 \\
\hline Lip, oral cavity and pharynx & $0.6(0.9)$ & $0.6(1.0)$ & -6.8 & 0.413 \\
\hline Head/brain & $0.4(1.0)$ & $0.5(1.0)$ & -7.4 & 0.171 \\
\hline
\end{tabular}

Data are mean (standard deviation) unless otherwise indicated

$p$ values were obtained using Wilcoxon tests

\section{Discussion}

\section{Main findings}

This retrospective study conducted in general and specialized practices in Germany revealed that the number of patients newly diagnosed with cancer per practice was lower during the COVID-19 (i.e., April 2020-March 2021) than during the pre-COVID-19 era (i.e., April 2019-March 2020). Interestingly, this decrease was observed for all practices, and ranged from $-6.6 \%$ in urology practices to -12.3 in dermatology practices. In addition, the decrease in the number of patients newly diagnosed with cancer per practice affected almost all sex and age categories, although this decreasing trend was significant only in a few groups. Finally, the strongest reduction in the number of cases was observed for skin cancers. To the best of the authors' knowledge, this is one of the first studies to have investigated not only the short-term but also the mediumterm effects of the COVID-19 pandemic on cancer diagnosis, while it is one of the largest studies on this topic.

\section{Interpretation of findings}

In the past months, a substantial body of literature has investigated the effects of the COVID-19 crisis on cancer diagnosis (Andrew et al. 2021; De Luca et al. 2021; Erdmann et al. 2021; Gurney et al. 2021; Jacob et al. 2021; Kuzuu et al. 2021; Patt et al. 2020; Peacock et al. 2021; 
Piontek et al. 2021; Reichardt et al. 2021; Ruiz-Medina et al. 2021; Stang et al. 2020; Tsibulak et al. 2020; Vardhanabhuti and $\mathrm{Ng}$ 2021; Voigtländer et al. 2021). For example, a study, using national data from New Zealand, showed that cancer registrations (new diagnoses of primary malignant cancers) declined by $40 \%$ in this country in March-April 2020 compared with 2018-2019, and that registrations increased in the subsequent months to reach pre-lockdown levels (Gurney et al. 2021). This decrease in cancer registrations was associated with a decline in endoscopies and radiation therapy attendances. In another study conducted at two hospitals in Spain, there was a $17 \%$ decrease in cancer diagnoses in 2020 compared with 2019, and the most impacted cancers were central nervous system, kidney and prostate neoplasms (Ruiz-Medina et al. 2021). These findings were corroborated in a third study from Belgium, as there was a $44 \%$ reduction in the diagnoses of invasive cancers in this country in April 2020 compared with April 2019 (Peacock et al. 2021). Similar results emerged from the majority of studies conducted in Germany at the early stage of the COVID-19 pandemic (Erdmann et al. 2021; Piontek et al. 2021; Reichardt et al. 2021; Stang et al. 2020; Voigtländer et al. 2021). A study from Saxony revealed that the number of new cases of cancer decreased in this state between January and September 2020 compared with the same period in 2017-2019 (Piontek et al. 2021), while another study, using data collected in North Rhine-Westphalia, reported a decrease in new cancer cases in January-September 2020 compared with 2019 (Stang et al. 2020). The present study adds to the literature on this topic by showing that the COVID19 not only had deleterious effects on the diagnosis of cancer in the first few months of the pandemic but also on the whole year following the beginning of this health crisis. Another interesting finding of this study conducted in Germany is that skin cancers were the cancers the most impacted by COVID-19, and this result is in line with previous research. Indeed, a study of data obtained in the United Kingdom identified a $69 \%$ decrease in skin cancer diagnoses in March-June 2020 compared with the same period in 2019 (Andrew et al. 2021). Another Canadian study found that, after the beginning of the COVID-19 pandemic, biopsies dropped by $15 \%$ for all skin lesions, $18 \%$ for keratinocyte carcinoma, and $27 \%$ for melanoma (Asai et al. 2021). Based on the findings of the present study, there was not only a decrease in skin cancer diagnosis in the first months of the pandemic but in the whole year following the first lockdown. As a matter of fact, in Germany, general practitioners and dermatologists play a major role in the screening of skin cancers (Anders et al. 2017), and this screening may have been disrupted by the COVID-19 pandemic, resulting in a decrease in the number of patients newly diagnosed with skin cancers per practice in this country.

Two factors may explain the decrease in cancer diagnoses since the beginning of the outbreak of the COVID19 pandemic. First, the COVID-19 crisis has negatively impacted the activity of general and specialized practices. For example, a survey of approximately 530 general practitioners from Ireland revealed that the number of face-to-face consultations went from 32,160 in February 2020 to 10,484 in June 2020 (Homeniuk and Collins 2021). Another study including 678 dermatologists surveyed between April and May 2020 showed that daily clinical activity had reduced by more than $75 \%$ during the pandemic for almost half of the sample, while approximately $40 \%$ of participants reported working fewer days per week compared with before the COVID-19 pandemic (Conforti et al. 2021). The COVID-19 pandemic not only has resulted in a decrease in the activity of general and specialized practices, but has also impaired the coordination between different types of practices, and this has led to decreased referral for possible cancer diagnoses to specialized practices or hospital departments (Gathani et al. 2021; Maganty et al. 2021). Second, patients' fear and concerns about the COVID-19 might have favored delayed or avoided medical care. Interestingly, it was estimated in a study, including 4975 adults living in the United States, that around $41 \%$ of them had avoided medical care because of concerns about COVID-19, with $12 \%$ of the sample avoiding emergency care and 32\% routine care (Czeisler et al. 2020). In addition, another body of research conducted in Italy reported 12 pediatric cases of delayed access to hospital care in March 23-27, 2020, and all these cases were, at least partially, explained by the fear of parents to be infected with SARS-CoV-2 (Lazzerini et al. 2020).

\section{Public health implications and directions for future research}

Based on the findings of this study, the COVID-19 pandemic has negatively impacted cancer diagnoses in general and specialized practices in Germany. This disruption in cancer care may have major deleterious effects among patients with cancer, and these effects may include increased tumor dimensions (Vanni et al. 2021) and higher rates of node invasion at diagnosis (Linck et al. 2021) as well as higher shortterm (Lai et al. 2020) and long-term mortality (Alagoz et al. 2021; Malagón et al. 2021). Public health measures should be implemented in this country to mitigate the deleterious effects of this health crisis on the diagnosis of cancer. Teleconsultations may be an alternative to traditional face-toface consultations in cancer care (Elkaddoum et al. 2020; Kjeldsted et al. 2021), and these teleconsultations may help identifying patients with symptoms requiring additional investigation (e.g., skin lesion with irregular border, recent 
breast mass and rectal bleeding). Another important measure is the education of the general public about the COVID-19 to reduce patients' fear of infection with SARS-CoV-2, and to encourage patients, despite the ongoing pandemic, to consult their primary care physician either when symptoms are present or for scheduled follow-up consultations. Finally, in terms of future research, more studies are warranted to analyze the medium-term impact of the COVID-19 pandemic on cancer diagnosis in other settings and countries. Besides, further research is also needed to better characterize the factors involved in the decreased cancer diagnoses during the COVID-19 era. More specifically, future studies should investigate the exact role of the overwhelming of healthcare systems and the fear of patients to consult primary care practices in the decrease in the number of patients newly diagnosed with cancer since the beginning of the COVID19 pandemic.

\section{Strengths and limitations}

The strengths of this study are the large number of general and specialized practices included in the analyses, and the use of data collected until March 2021. However, the study results should also be interpreted in the light of several limitations. First, this study included practices only, and it was, therefore, not possible to analyze the effects of the COVID19 pandemic on cancer diagnosis at hospital. Besides, not all practices were included in the analyses, although some of these practices (e.g., hematology and oncology practices) are actively involved in the diagnosis of cancers in Germany. Second, cancer diagnoses were documented using ICD-10 codes, and more clinical data (e.g., type of symptoms, delay between the onset of symptoms and the diagnosis of cancer, and cancer staging at diagnosis) may have allowed more detailed analyses. Third, there was no information on major sociodemographic characteristics (e.g., employment, income and health insurance status), and the impact of the COVID19 crisis on cancer diagnosis in specific sociodemographic groups could not be studied.

\section{Conclusions}

This study, including more than 1400 general and specialized practices from Germany, found a decrease in the number of patients newly diagnosed with cancer in April 2020-March 2021 compared with April 2019-March 2020. This decrease was observed in all practices and in most of sex and age groups. In addition, the study revealed that skin cancers were the cancers the most impacted by the COVID19 pandemic. More research is warranted to corroborate or invalidate these findings in other settings, while it is of utmost importance to better understand the role played by the overwhelming of healthcare systems and fear of patients in this decrease in cancer diagnoses.

Author contributions LJ contributed to the design of the study, managed the literature searches, wrote the first draft of the manuscript, and corrected the manuscript. MK contributed to the design of the study and corrected the manuscript. KK contributed to the design of the study, performed the statistical analyses, and corrected the manuscript. All authors contributed to and have approved the final manuscript.

Funding This research did not receive any specific grant from funding agencies in the public, commercial, or not-for-profit sectors.

Data availability The data that support the findings of this study are available from the corresponding author upon reasonable request.

\section{Declarations}

Conflict of interest The authors declare no competing financial interests.

Ethical approval and consent to participate German law allows the use of anonymous electronic medical records for research purposes under certain conditions. According to this legislation, it is not necessary to obtain informed consent from patients or approval from a medical ethics committee for this type of observational study that contains no directly identifiable data.

\section{References}

Alagoz O, Lowry KP, Kurian AW et al (2021) Impact of the COVID19 pandemic on breast cancer mortality in the US: estimates from collaborative simulation modeling. J Natl Cancer Inst 113:14841494. https://doi.org/10.1093/jnci/djab097

Anders MP, Fengler S, Volkmer B et al (2017) Nationwide skin cancer screening in Germany: evaluation of the training program. Int $\mathbf{J}$ Dermatol 56:1046-1051. https://doi.org/10.1111/ijd.13688

Andrew TW, Alrawi M, Lovat P (2021) Reduction in skin cancer diagnoses in the UK during the COVID-19 pandemic. Clin Exp Dermatol 46:145-146. https://doi.org/10.1111/ced.14411

Asai Y, Nguyen P, Hanna TP (2021) Impact of the COVID-19 pandemic on skin cancer diagnosis: a population-based study. PLoS ONE 16:e0248492. https://doi.org/10.1371/journal.pone.0248492

Bundesärztekammer (2021) Bundesärztekammer. https://www.bunde saerztekammer.de/. Accessed 23 Dec 2021

Conforti C, Lallas A, Argenziano G et al (2021) Impact of the COVID19 pandemic on dermatology practice worldwide: results of a survey promoted by the international dermoscopy society (IDS). Dermatol Pract Concept 11:e2021153. https://doi.org/10.5826/ dpc.1101a153

Czeisler MÉ, Marynak K, Clarke KEN et al (2020) Delay or avoidance of medical care because of COVID-19-related concerns-United States. MMWR Morb Mortal Wkly Rep 69:1250-1257. https:// doi.org/10.15585/mmwr.mm6936a4

De Luca P, Bisogno A, Colacurcio V et al (2021) Diagnosis and treatment delay of head and neck cancers during COVID-19 era in a tertiary care academic hospital: what should we expect? Eur Arch Otorhinolaryngol. https://doi.org/10.1007/s00405-021-06834-1

Elkaddoum R, Haddad FG, Eid R, Kourie HR (2020) Telemedicine for cancer patients during COVID-19 pandemic: between threats 
and opportunities. Future Oncol 16:1225-1227. https://doi.org/ 10.2217/fon-2020-0324

Erdmann F, Wellbrock M, Trübenbach C et al (2021) Impact of the COVID-19 pandemic on incidence, time of diagnosis and delivery of healthcare among paediatric oncology patients in Germany in 2020: evidence from the German childhood cancer registry and a qualitative survey. Lancet Reg Health Eur 9:100188. https://doi. org/10.1016/j.lanepe.2021.100188

Gathani T, Clayton G, MacInnes E, Horgan K (2021) The COVID-19 pandemic and impact on breast cancer diagnoses: what happened in England in the first half of 2020. Br J Cancer 124:710-712. https://doi.org/10.1038/s41416-020-01182-z

Gurney JK, Millar E, Dunn A et al (2021) The impact of the COVID19 pandemic on cancer diagnosis and service access in New Zealand-a country pursuing COVID-19 elimination. Lancet Reg Health West Pac 10:100127. https://doi.org/10.1016/j.lanwpc. 2021.100127

Homeniuk R, Collins C (2021) How COVID-19 has affected general practice consultations and income: general practitioner crosssectional population survey evidence from Ireland. BMJ Open 11:e044685. https://doi.org/10.1136/bmjopen-2020-044685

Jacob L, Loosen SH, Kalder M et al (2021) Impact of the COVID-19 pandemic on cancer diagnoses in general and specialized practices in Germany. Cancers (basel) 13:408. https://doi.org/10.3390/cance rs 13030408

Johnson KD, Harris C, Cain JK et al (2020) Pulmonary and extrapulmonary clinical manifestations of COVID-19. Front Med (lausanne) 7:526. https://doi.org/10.3389/fmed.2020.00526

Kjeldsted E, Lindblad KV, Bødtcher $\mathrm{H}$ et al (2021) A population-based survey of patients' experiences with teleconsultations in cancer care in Denmark during the COVID-19 pandemic. Acta Oncol 60:1352-1360. https://doi.org/10.1080/0284186X.2021.1956688

Kuzuu K, Misawa N, Ashikari K et al (2021) Gastrointestinal cancer stage at diagnosis before and during the COVID-19 pandemic in Japan. JAMA Netw Open 4:e2126334. https://doi.org/10.1001/ jamanetworkopen.2021.26334

Lai AG, Pasea L, Banerjee A et al (2020) Estimated impact of the COVID-19 pandemic on cancer services and excess 1-year mortality in people with cancer and multimorbidity: near real-time data on cancer care, cancer deaths and a population-based cohort study. BMJ Open 10:e043828. https://doi.org/10.1136/bmjop en-2020-043828

Lantelme P, Couray Targe S, Metral P et al (2020) Worrying decrease in hospital admissions for myocardial infarction during the COVID-19 pandemic. Arch Cardiovasc Dis 113:443-447. https:// doi.org/10.1016/j.acvd.2020.06.001

Lazzerini M, Barbi E, Apicella A et al (2020) Delayed access or provision of care in Italy resulting from fear of COVID-19. Lancet Child Adolesc Health 4:e10-e11. https://doi.org/10.1016/S23524642(20)30108-5

Linck P-A, Garnier C, Depetiteville M-P et al (2021) Impact of the COVID-19 lockdown in France on the diagnosis and staging of breast cancers in a tertiary cancer centre. Eur Radiol. https://doi. org/10.1007/s00330-021-08264-3

Maganty A, Yu M, Anyaeche VI et al (2021) Referral pattern for urologic malignancies before and during the COVID-19 pandemic. Urol Oncol 39:268-276. https://doi.org/10.1016/j.urolonc.2020. 11.027

Malagón T, Yong JHE, Tope $\mathrm{P}$ et al (2021) Predicted long-term impact of COVID-19 pandemic-related care delays on cancer mortality in Canada. Int J Cancer. https://doi.org/10.1002/ijc.33884
Patt D, Gordan L, Diaz M et al (2020) Impact of COVID-19 on cancer care: how the pandemic is delaying cancer diagnosis and treatment for American seniors. JCO Clin Cancer Inform 4:1059-1071. https://doi.org/10.1200/CCI.20.00134

Peacock HM, Tambuyzer T, Verdoodt F et al (2021) Decline and incomplete recovery in cancer diagnoses during the COVID-19 pandemic in Belgium: a year-long, population-level analysis. ESMO Open 6:100197. https://doi.org/10.1016/j.esmoop.2021. 100197

Piontek D, Klagges S, Schubotz B et al (2021) Documented new cases of cancer in the clinical cancer registries of the German state of Saxony during the COVID-19 pandemic. Dtsch Arztebl Int 118:328-329. https://doi.org/10.3238/arztebl.m2021.0216

Rathmann W, Bongaerts B, Carius H-J et al (2018) Basic characteristics and representativeness of the German disease analyzer database. Int J Clin Pharmacol Ther 56:459-466. https://doi.org/10. $5414 / \mathrm{CP} 203320$

Reichardt P, Bollmann A, Hohenstein S et al (2021) Decreased incidence of oncology admissions in 75 Helios hospitals in Germany during the COVID-19 pandemic. Oncol Res Treat 44:71-75. https://doi.org/10.1159/000512935

Ruiz-Medina S, Gil S, Jimenez B et al (2021) Significant decrease in annual cancer diagnoses in Spain during the COVID-19 pandemic: a real-data study. Cancers (basel) 13:3215. https://doi.org/ 10.3390/cancers 13133215

Stang A, Kühling L, Khil L et al (2020) Drop in cancer reporting by pathologists in North Rhine-Westphalia, Germany, during the COVID-19 lockdown. Dtsch Arztebl Int 117:886-887. https:// doi.org/10.3238/arzteb1.2020.0886

Tangcharoensathien V, Bassett MT, Meng Q, Mills A (2021) Are overwhelmed health systems an inevitable consequence of covid-19? Experiences from China, Thailand, and New York state. BMJ 372:n83. https://doi.org/10.1136/bmj.n83

Tsibulak I, Reiser E, Bogner G et al (2020) Decrease in gynecological cancer diagnoses during the COVID-19 pandemic: an Austrian perspective. Int J Gynecol Cancer 30:1667-1671. https://doi.org/ 10.1136/ijgc-2020-001975

Uchino K, Kolikonda MK, Brown D et al (2020) Decline in stroke presentations during COVID-19 surge. Stroke 51:2544-2547. https:// doi.org/10.1161/STROKEAHA.120.030331

Vanni G, Pellicciaro M, Materazzo M et al (2021) Advanced stages and increased need for adjuvant treatments in breast cancer patients: the effect of the one-year COVID-19 pandemic. Anticancer Res 41:2689-2696. https://doi.org/10.21873/anticanres.15050

Vardhanabhuti V, Ng KS (2021) Differential impact of COVID-19 on cancer diagnostic services based on body regions: a public facility-based study in Hong Kong. Int J Radiat Oncol Biol Phys 111:331-336. https://doi.org/10.1016/j.ijrobp.2021.05.010

Voigtländer S, Hakimhashemi A, Inwald EC et al (2021) The impact of the COVID-19 pandemic on cancer incidence and treatment by cancer stage in Bavaria, Germany. Dtsch Arztebl Int 118:660-661. https://doi.org/10.3238/arztebl.m2021.0329

Wiersinga WJ, Rhodes A, Cheng AC et al (2020) Pathophysiology, transmission, diagnosis, and treatment of coronavirus disease 2019 (COVID-19): a review. JAMA 324:782-793. https://doi. org/10.1001/jama.2020.12839

World Health Organization (2021) WHO coronavirus (COVID-19) dashboard. https://covid19. who.int/. Accessed 23 Dec 2021

Publisher's Note Springer Nature remains neutral with regard to jurisdictional claims in published maps and institutional affiliations. 